\title{
Application of Three-Dimensional Geological Modelling in Coal Mining
}

\author{
Liyan Ren ${ }^{1,2^{*}}$, Huayang Dai ${ }^{3}$, Yingcheng $\mathrm{Li}^{1,2,4}$ and Enquan Wang ${ }^{1,2}$ \\ ${ }^{1}$ Key Laboratory for Aerial Remote Sensing Technology of National Administration of Surveying, Mapping and \\ Geoinformation, Beijing, China \\ ${ }^{2}$ China TopRS Technology Co. Ltd., Beijing, China \\ ${ }^{3}$ College of Geoscience and Surveying Engineering, China University of Mining and Technology (Beijing), Beijing, China \\ ${ }^{4}$ Chinese Academy of Surveying and Mapping, Beijing, China
}

\begin{abstract}
Three-dimensional geological modelling of coal mines is critical to the sustainable development of the mining industry. On the basis of comprehensively analyzing 3D geological modelling methods, according to the available data of mine such as geological terrain maps, cross-sections and boreholes, we present a 3D modelling method by integrating ArcGIS and 3D GeoModeller. Our motivation is to integrate the advantages of both software modules in processing, converting, integrating and transferring geological information. Special attention has been given to the data structure and processing flow. We build a 3D geological model of the Xieqiao coal mine and successfully extract the geological framework of the strata in the 11118 workface, which is consistent with that of the actual explored geology.
\end{abstract}

Keywords: 3D geological modelling, integrated system, Geodatabase, 3D Geomodeler, ArcGIS

\section{Introduction/Background}

Pertinent impediments such as a lower information degree, the out-of-date information infrastructure and the low level of information management and decision-making make it critical for there to be improvements in mines. Modern exploration technologies favour the construction of digital mine. Digital mine promotes the transformation of the mining industry by integrating information, automation and intelligence to build safe, efficient, high-yield, green and sustainable mines.

Three-dimensional geological modelling and visualization of mines are at the core of digital mine. Compared with traditional 2D geological data, 3D models express the various geological phenomena completely, as well as reproduce the spatial distribution of geological units quickly, allowing for the prediction of mining subsidence, better decision-making and improved geological analysis.

Modelling methods such as multi-DEM, tetrahedral grid and the generalized tri-prism that are based on borehole estimation and interpolation data are used to construct 3D models of mine geology (Chen 1995, Wang et al 2003, Wu 2004a); nevertheless, these methods cannot fully use multisource mining data, whereas multi-source integration methods can be used to build 3D models (Kaufmann and Martin 2008, Zhao et al 2009) but require user interaction in model construction and low automation in model updates.

During coal production, a plethora of geological data is accumulated. The organisation of discreteinformation under dynamic conditions and the extraction of meaningful information to better service for three-dimensional modelling is a meaningful work (Wu et al 2004b).

In this study, we comprehensively analyse 3D geological modelling methods and the characteristics of the coal industry and present a methodology for constructing 3D models of mine geology by integrating ArcGIS and 3D GeoModeller (Mcinerney 2014). The latter is 3D geological modelling software developed by BRGM, the Geological Survey of France, and commercialized by Intrepid Geophysics. The model construction allows for quick generation of different interpretations and addition of new data, which summarizes the geology by implicit 3D potential field. However, the importing of data files must follow a specific format; therefore, pre-processing of raw data is required prior to $3 \mathrm{D}$ modelling. ArcGIS is powerful in terms of organising and analysing data in space. The proposed integrated system fully utilizes the advantages of both systems and considers geological terrain maps, crosssections and borehole data. We tested the proposed method by constructing a 3D geological model of the Xieqiao coal mine in Huainan, China.

\section{Integrated System and Data}

\subsection{Data sources}

The main geological data available in mine can be divided into two types: one is the interpretation information, such as geological terrain maps and cross-sections. They are the projection of the real three-dimensional orebody onto a plane (XY plane, XZ plane, or YZ plane). Geological terrain maps show the topography, surface features and outcrops. These data are used to covey topographic information and

* Corresponding Author: L.Y. Ren, swallow.ren@163.com, phone: +86 15210572391

Copyright (C) 2017 Canamaple Academia Services, http://press.camdemia.ca

DOI: $10.15273 /$ gree.2017.02.040 
the boundary distribution of outcrops, but they cannot represent the formations. To overcome this limitation, crosssections are used to offer data interpretation in the vertical direction. The other type of data is field survey records that contain structural information and borehole data described with stratigraphic, lithological and inclinometer information. All datasets are typically large and in non-digital form, obtained at different times, different scales and coordinates.

\subsection{Principles of 3D geological modelling}

Regionalized variables are a basic concept ingeostatistics. A point is defined in $3 \mathrm{D}$ space as $P=(x, \mathrm{y}, \mathrm{z}) . T(p)$ is a scalar function of point $P$; thus, $T(p)$ is a regionalised variable that is often highly variable and discontinuous; therefore, it cannot be directly studied or described by an ordinary function, and it is typically studied in an increment manner for acquiring the overall structure. The properties of geological bodies are regionalised variables. Their basic features are the continuous change and anisotropy in space, and the value of a point in space is related to the values of the adjacent points in certain ranges. The 3D GeoModeller (Intrepid Geophysics) (Zengerer 2014) uses geological contact locations and orientation data from formation as raw data. Both types of data are cokriged to interpolate the continuous 3D potential field that describes the geometry of the geological bodies; the geological boundaries are extracted as isopotential surfaces. The topological relations among geological bodies are defined using geological piles that contain the geological history. Based on the above concepts, the potential incremental $T^{*}(p)-T^{*}\left(p_{0}\right)$ is formulated as follows:

$$
T^{*}(p)-T^{*}\left(p_{0}\right)=\sum_{\alpha=1}^{M} \mu_{\alpha}\left(T\left(p_{\alpha}\right)-T\left(p_{\alpha}^{\prime}\right)\right)+\sum_{\beta=1}^{N} v_{\beta} \frac{\partial T}{\partial u_{\beta}}\left(p_{\beta}\right)
$$

where $p_{0}$ is a fixed arbitrary initial point, $p$ is any point, $\mu_{\alpha}$ and $v_{\beta}$ are weights that are functions of $p$ and are determined by the co-kriging system, and $T\left(p_{\alpha}\right)$ and $T\left(p_{\alpha}^{\prime}\right)$ are potential values at the same interface; the right half-side of the equation is the gradient of the potential field, taken from the polarization unit vector of the formation.

This method describes the 3D geological space by formulating the potential field in which the geological boundaries are isopotential surfaces and their dips are represented as gradients of the potential. The geological contacts are digitised from the cross-sections. The geometry of the geological bodies is obtained by discrediting the reference isovalues. The scalar functions representing the discrete geological series are automatically merged to build the 3D model (Calcagno et al 2006, Calcagno et al 2008, Chilès et al 2004, Ming et al 2010).

\subsection{Integrated system}

The architecture of the integrated system is shown in Figure 1. First, all mining data are stored in Geodatabase of ArcGIS. Then, the spatial data are presented as points, polylines and polygons, which are efficiently managed, organized and extracted. Next, the shp data are imported to ArcGIS and analysed prior to building the 3D geological model. Finally, the shp and mif files are converted and exchanged into 3D GeoModeller files to build the 3D geological model. Finally, 2D profile data are re-introduced into ArcGIS and established the corresponding feature class in the Geodatabase. The data processing flow is shown in Figure 2.

First, the data are classified into geological terrain maps, cross-sections and borehole data. The data are in AutoCAD format.

Second, the various types of data are processed respectively. One is to re-position these data by an independent coordinate in mine to ensure all data with common reference system and consistent spatial topological relations. For example, the end of cross-section must be as the starting position of profile, and then the points on crosssection are repositioned. Two is to digitise these data and then extracted the useful information. From geological terrain maps, the surface elevation points and boundaries of outcrops are extracted. From cross-sections, the interfaces of coal seams and main stratums are extracted. From boreholes, the immediate roof and main roof are extracted and the corresponding stratum pile is established. Three is to establish information tables of location, inclinometer and lithologic.

Third, the points and lines are digitised and extracted as shp files, to ArcGIS. All data are structured by combining boreholes. Then, a database based on Geodatabase is built, and the data are stored as points and lines with attributes.

Fourth, the data are checked, selected and analysed. Geostatistics are used to analyse coal seam information from the borehole data, and 2D analysis data for coal seam thickness, structure and variations are added to the 3D model.

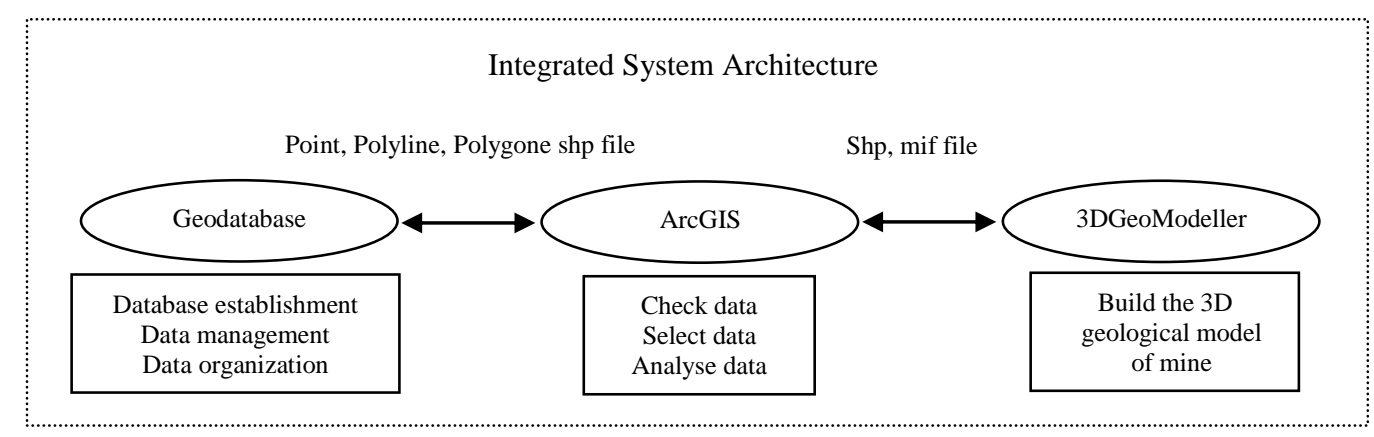

Figure 1. Integrated system architecturecomponents: the communication among the different software module is based on files; the respective functions are also shown. 


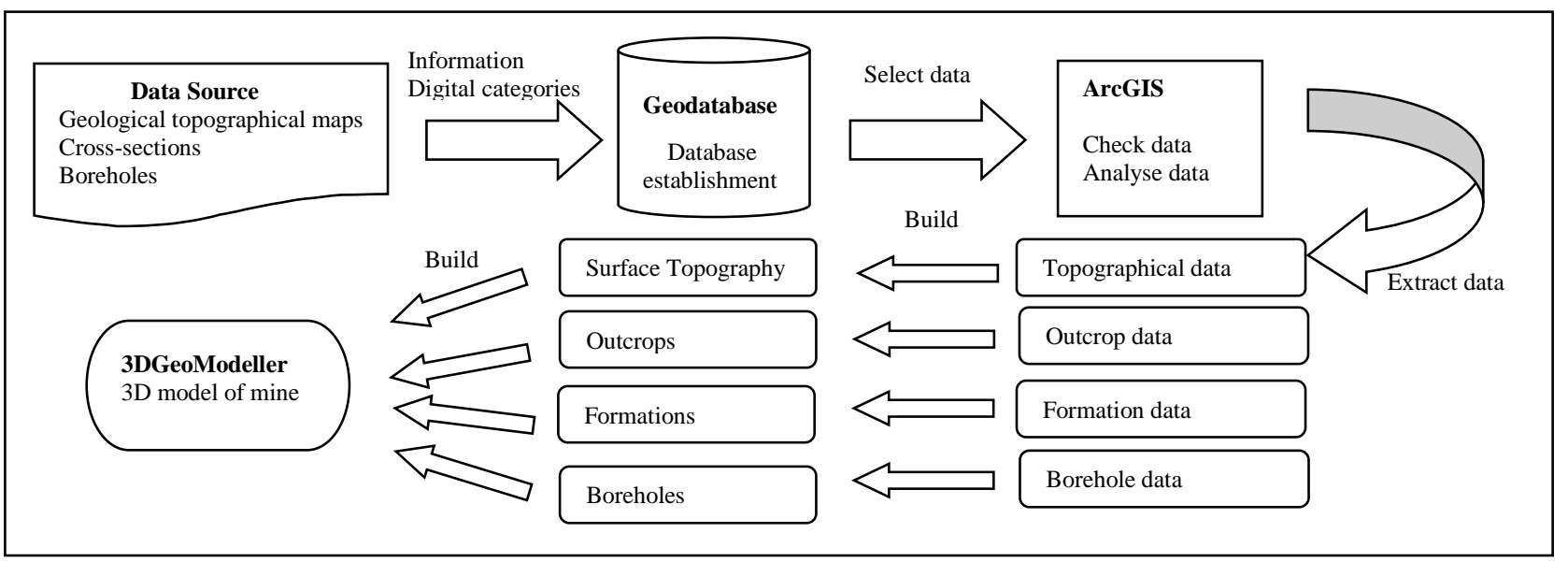

Figure 2. Processing flows.

Fifth, the surface points are used to generate the digital terrain model (DTM) surface using kriging for modelling the surface topology. The boundary lines and attitudes of the outcrops are used to build the 3D model of the outcrops. The cross-sections are digitised as objects with attributes and are combined with orthogonal data to establish the main formations. Then we build the 3D geological model of the mine.

Sixth, based on the location of the boreholes, inclinometer information is used to build borehole trajectory in the 3D model. The model is verified and corrected, with checking whether the borehole is detected on the corresponding layer, and fixing the ground contact surface. Each entity is created using stratigraphic grid formation, and then 3D formation of regional solid model is generated. In addition, new exploration data are added to update the database for the model update.

\section{Case Study}

\subsection{Study area, data, object}

We used the proposed method to analyse and model the Xieqiao coal mine in Huainan, China. The study area is shown in Figure 3. It is in the Xieqiao coal mine and includes the subsidence range of 11118 workface. There are no faults in the region. Constructions of the 3D geological model in the area and extraction of the geological conditions overlying strata upon the 11118 work-face are important for subsidence prediction.

The data for the modelling include geological terrain map at 1:5000 scale, 7cross-sections at 1:2000 scale and 13 boreholes. The main roof and immediate roof data are acquired using borehole column maps. Then the 3D model of 11118 work-face is constructed from old to young mainlybasements, 8 coal seams, immediate roof, main roof, overly strata, tertiary system, quaternary system, and surface topography.

\subsection{Application and results}

The geographic database comprises all the data needed in the modelling. The data are processed through above methodology. Validated data from the study area were selected for the modelling.

An independent coordinate system was adopted. A project was created by defining its limits $2.5 \mathrm{~km} \times 2 \mathrm{~km} \times$ $1.1 \mathrm{~km}$ are shown in Figure 4. The model area extends from $43000 \mathrm{~m}$ to $45500 \mathrm{~m}$ in the X-direction (West to East), 28500 to $30500 \mathrm{~m}$ in the Y-direction (South to North) and from $-900 \mathrm{~m}$ to $200 \mathrm{~m}$ in the Z-direction. The surface topography was modelled from a DTM using a $50 \mathrm{~m} \times 50$ $\mathrm{m}$ grid. Cross-sections were created, and geological data were imported. The 3D model was computed and rendered in 2D, and the boreholes were incorporated.

Main geological results derived from the modelling are (i) the overall 3D geological conditions cover 11118 wok-face are shown. The relationship of formations is presented. The model comprised of 8 geology formation the order see in study object above. And the model shows that local terrain is flat; area is covered by thick alluvium. (ii) This kind of model produces a consistent representation of subsurface geology that may be a support for being compatible with finite element software such as FLAC $^{3 \mathrm{D}}$. (iii) The cross-section passing 11118 work-face was extracted which is shown in Figure 5.

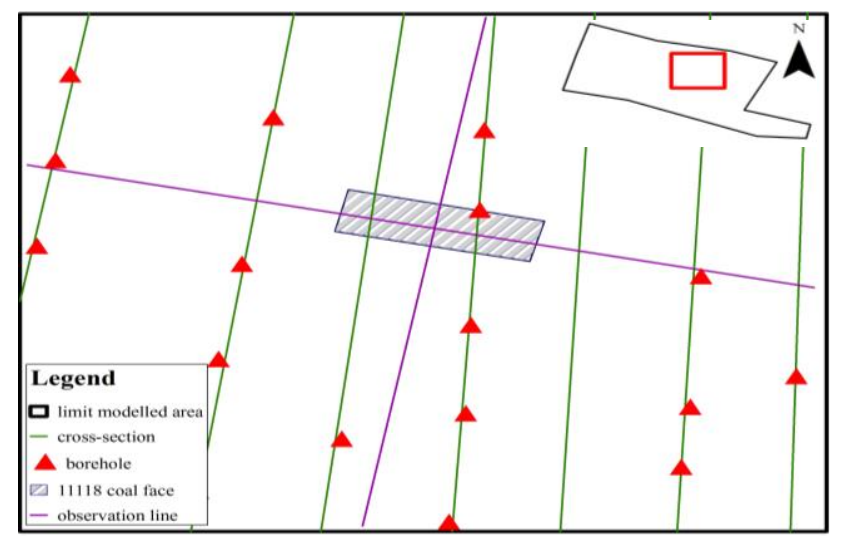

Figure 3. Simplified geological map of the study area in the Xieqiao coal mine. The boundaries of the mine and study area are shown inthetop right corner. 


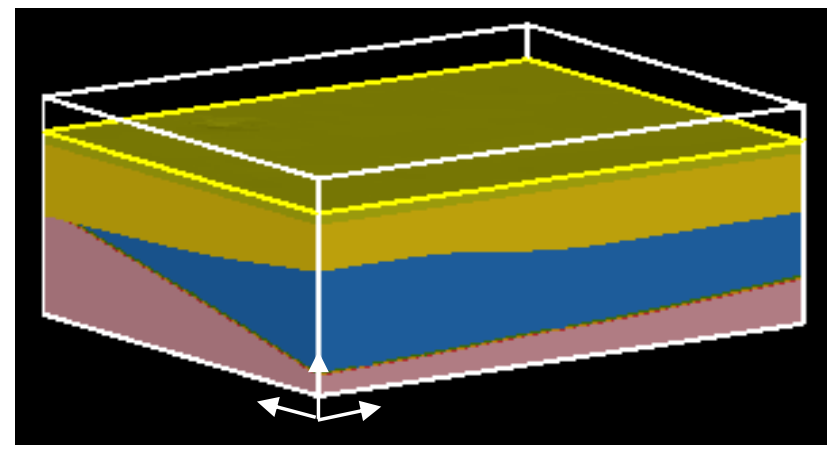

Reference: Bottom

Quaternary_System_Seri... (Onlap)

Tertiary_System_Series (Erode)

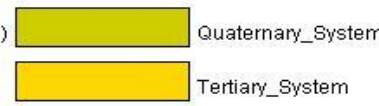

Rockandcoal_Series (Onlap)

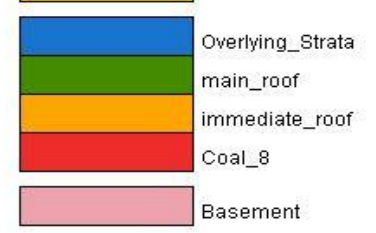

Basement_Series (Onlap)

Figure 4. 3D geological model of the study area.

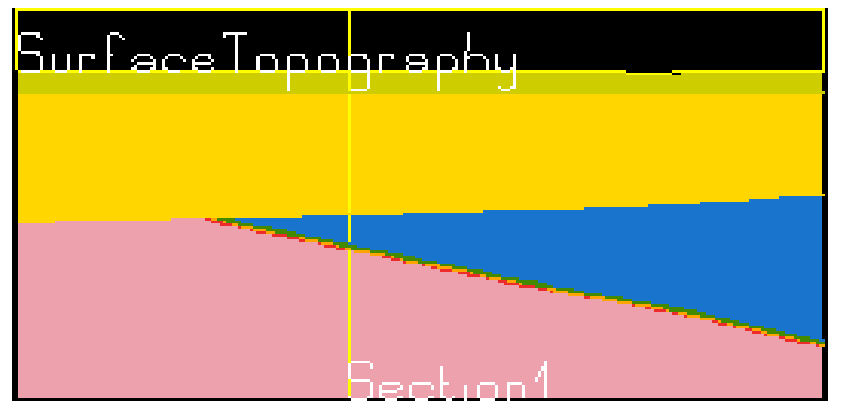

Figure 5. Cross-section of the 11118 workface extracted from 3D model.

\section{Conclusions}

In this work, we present an integrated system for modelling 3D geology of mine on the platform of ArcGIS and 3D GeoModeller, and describe the processing flows in detail. The newer method is able to take account more types of data obtained in mine (geological terrain maps, cross-sections and boreholes) constructing 3D geological model.

The proposed integrated system is well suited for 3D geological modelling of mines. It offers intelligent data management and processing, efficient data checking, selection and analysis, automated model construction and updating.

\section{Acknowledgement}

This work is jointly supported by projects 2016YFC0803109 and 2016YFC0803104 of National Key Research and Development Program. The authors would like to express appreciations to Intrepid Geophysics and BRGM for their 3D GeoModeller software (evaluation license). We also thank Dr. Bin Liu for his helpful advice on data processing. All data were provided by Xieqiao coal mine.

\section{References}

Calcagno, P., G. Courrioux, A. Guillen, D. Fitzgerald and P. Mcinemey, 2006. How 3D implicit geometric modelling helps to understand geology: the 3DGeoModeller methodology. Proc. Society for Mathematical Geology 11th International Congress, Université de Liège, Belgium.

Calcagno, P., J.P. Chilès, G. Courrioux and A. Guillen, 2008. Geological modelling from field data and geological knowledge Part I. Modelling method coupling 3D potential-field interpolation and geological rules. Physics of the Earth and Planetary Interiors, 17(1): 147 - 157.

Chen, X.Y., 1995. A workstation for three-dimensional spatial data research. The 4th International Symposium of LIESMARS, Wuhan, China, 42 - 51.

Chilès, J.P., C. Aug, A. Guillen and T. Lees, 2004. Modelling the geometry of geological units and its uncertainty in 3D from structural data: the potentialfield method. International Symposium on Orebody Modelling and Strategic Mine Planning, Perth, Australia, 313 - 320.

Mcinerney P., 2014. GeoModeller tutorial B: Using drillholes in 3D GeoModeller-a model-building strategy. $\quad$ http://www.intrepidgeophysics.com/ig/index.php?page=downloadgeomodeller-tutorials.

Kaufmann, O. and T. Martin, 2008. 3D geological modelling from boreholes, cross-sections and geological maps, application over former natural gas storages in coal mines. Computers and Geosciences, 34(3): 278 - 290.

Ming, J., M. Pan, H.G. Qu and Z.H. Ge, 2010. GSIS: A 3D geological multi-body modelling system from netty cross-sections with topology. Computer and Geosciences, 36(6): 756 - 767.

Wang, C.X., S.W. Bai and H.J. He, 2003. Study on geological modelling in 3D strata visualization. Chinese Journal of Rock Mechanics and Engineering, 22(10): 1722 - 1726.

$\mathrm{Wu}, \mathrm{L} . X ., 2004 \mathrm{a}$. Topological relations embodied in a generalized tri-prism(GTP) model for a 3D geoscience modelling system. Computers and Geosciences, 30(4): 405 - 418.

Wu, L.X., W.X. Zhu and R.X. Zhang, 2004b. Digital mine and the future development of mines in China. Mining Science and Technology, 7: 29 - 31.

Zengerer, M., 2014. Use of geostatistically-constrained potential field inversion and downhole drilling to predict distribution of sulfide and uranium mineralisation. The Ninth International Mining Geology Conference, Adelaide, Australia, 281 - 289.

Zhao, X.D., L.G. Li and L.J. Peng, 2009. GIS and GOCAD-based methodology of 3D geological modelling of mine and its application. Geography and Geo-Information Science, 25(2): 34 - 38. 